\title{
EPIGRAMMATA GRAECA
}

\author{
EX LAPIDIBVS CONLECTA
}

EDIDIT

GEORGIVS KAIBEL.

\section{BEROLINI}

APVD G. REIMER.

1878. 



\title{
FRANCISCO BVECHELER
}

\section{HERMANNO VSENER}

\author{
V. S. L. M.
}



neque in bona regete nullwm eat opicum nequam neque in maka non aliquod bonsm.

Varro.

Vestrae fidei vestroque, viri optimi, amori quoniam hunc librum conmendaturus sum paucis exponere decet qualis quem ante hos sex annos nascentem vidistis singularique studio foristis iam adultus ad vos redeat.

Epigrammata graeca e lapidibus excepta triplex fuit munus conligendi emendandi adnotandi, quorum primum sane non aeque mibi fuit difficile et ambiguum quam eis qui olim similem rem adgressi non tam conlegerunt epigrammata quam elegerunt. Mibi vero cum omnium quotquot ex lapidibus descripta habentur epigrammatum graecorum non eclogen sed syllogen plenam fere et absolutam proponere consilinm fuerit, si quae desiderantur ea errore culpaque mea onjissa putaveritis. Consulto vero omisi cum ea quae adeo mutila erant at ne de natura quidem tituli integri indicari posset tum ea quae ex codicibus anthologiisve petita iniuria aliquamdiu inter titulos ferebantur, velut epigramma Hesiodi tripodi inscriptum, Homeri Sapphonis Diogenis Oppiani epitaphia, Pythiae denique oraculum (C. J. 1724) a Cyriaco ex codicibus transscriptum et a Moroniano ex H. Stephani editione interpolatum, ut taceam de Aureoli titulo vilissimo (C. J. 6761), quo tandem liberavit nos Mommseni prudens indicium (Anal. epigr. p. 255). Haec igitur cum non verendum sit ne quo invito praetermiserim, rectius fortasse ideo inculpabor quod ea non recepi quae etsi nemo recentioris memoriae in lapide viderit tamen vere inscripts fuisse aut luculentissimis constet testimoniis aut certissime demonstrari possit, velut quae in Anthologiae 
praefatio.

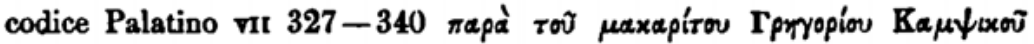
descripta leguntur quaeve cum alii scriptores tum Pausanias ex periegetarum fide haud ambigus rettulit. Quae omnia una cum oraculis aliisque similibus inscriptionibus genuinis singulari aliquando libro conplexus edam, neque minus quae nostrae aetatis antiquitates investigandi sedulitas reperiendique felicitas nova protulerit conligere et ex instituto consilio tractare pergam.

Inedita haud ita multa mihi praesto fuisse epigrammata ipsa res tulit. Nam etsi amicorum auxilio nunquam destitutus accipiebam fere quae sive Romae et Athenis sive in aliis Graeciae Italiaeve partibus reperiebantur, tamen feliciter factum est ut nostrates quae alicuius essent momenti ad veterum res litterasque cognoscendas quam primum fieri posset edere consuerint. Sunt vero quae primus ego prodam sive a me ipso olim descripta sive aliorum beneficio debita.

Difficilius erat conlecta generibus inlustrare partibusque apte discribere. Cum enim in unum conprehendenda essent quae decem fere saeculorum spatio in omnibus fere orbis terrarum partibus, ubi quidem sermo graecus vigeret, lapidibus mandata essent, non e re fore arbitrabar si, quod recte faciunt qui Inscriptionum Corpora condunt, ex solo geographico qui dicitur ordine epigrammsta digererem, qua scilicet re saepius disiungerentur quae iungenda erant diversaque plsne conmiscerentur, sed varia argumentorum genera distinguenda, praeteres temporum locorum ingenii disciplinae artis ratio habenda erst. Meliors enim quarto a. Chr. n. quam tertio p. Chr. n. saeculo proveniunt, meliora plerumque Athenis quam apud Macedones Syrosque, melius Graeci quam barbari cultae linguae sero adsueti locuntur, et quod gravissimum est in certis quibusdam civitatibus provinciisque certum quoddsm dicendi sentiendique genus florebat exercebaturque, quae omnes res, si quidem ipse mihi constare volui, in censum vel maxime erant vocsndae. Itaque ut de sepulcralibus primum dicam titulis primam partem atticoram feci, ita tamen ut quae graecae essent setatis quaeque romanorum temporum esse viderentur secernerem. Subiunxi insularum Asiseque epigrammata, in quibus indolis graecae sive mavis artis alexandrinae longissime patent vestigia et fructus. Sed earundem regionum cum longe maior pars inferiorum esset temporum et hominum incultiorum, priore loco posui melioris notae epigrammata, altero reliquorum multitudinem adieci provinciarum fere ordinem secutus: nsm in unum conponendi 
erant Lydo rum tituli, qui multi sunt et tamquam helluantur in varia de rebus futuris cogitatione (nescio an Erythraeis ducibus, beatorum colonis, cf. ad n. 904), neque minus Phrygiorum, quibus Graecorum de inferis fabulae maximam in contemptionem venerunt. Denique pecaliarem locum Syris adsignavi, quorum quae potissima scribendi ratio fuerit neminem fugiet legentem. Ex Asia in Graeciam remigravi, cui priores partes eam ob rem denegaram ne vilia infimae aetatis carmina quae praevalent elegantissimis Ionum Dorumque epigrammatis anteponere cogerer: quamquam hac de re num recte iudicaverim iamiam dubitationis aliquid subnascitur. Restant qui a Graecis didicerunt Romani, qui quam sollerter veterum epigrammatographorum exempla secuti sint et excoluerint et Anthologiae poetae plurimi docent et prior quam a reliquis scparavi Romanorum et Neapolitanorum epigrammatum pars: praevalent antem bic quoque ut par est viliora.

Dedicatoria longe diversum sibi postulabant ordinem, at quae et pauciora essent et pro argumentorum diversitate sponte in diversas discederent partes : nam et ephebica et agonistica inter se divisi (nec poenitet fecisse) et proscynemata hymnos oracula iure mihi videor dedicatoriis adnumerasse. Septem igitur institui partes, quarum quo quamque modo disposuerim explicare nihil adtinet.

Claudunt agmen epigrammata varia quattuor in partes divisa omnia, e quibus prima terminorum aedificiorum fontium pontium titulos, quarta amulets et diras conplectitur, reliquas duas magis dubito num recte administrarim.

Conlecta dum edo schedasque prelo trado haud mediocris succrevit Addendorum copia cum eorum quae recens inventa sunt tum eorum quae ego huiusmodi negotii nondum satis peritus vel omiseram vel falso olim loco conlocata retraxeram. Eisdem autem de causis vel Addendis quae adderem habui, quae in huius praefationis calce subicienda curavi; nonnulla doleo quod post confectos demum et typis expressos indices accesserunt. Addendis passim intermiscui corrigends; correxi tamen ea tantum quas legentes offendere viderentur, alia permulta sciens praetermisi. Quod incredibili operarum socordis et inperitia factum est ut innumeris fere locis spirituum accentuumque notae dilapsae abierint, aegre hoc fero, aegrius vero quod ipse justo saepius accentus perperam positos corrigere neglexi; qui errores quam digni sint qui graviter castigentur probe scio, quamquam neque seque neque prudenter existimantis 
iudicis est in eis solis indagandis aut tempus terere ant eruditionem ostentare.

Superest longe difficillimum emendandi adnotandique munus. Duae sutem sunt adnotationis partes, alters quae antecedit ipsum epigramma locum ubi repertum sit editoresque indicans : ubi minime omnium posui editorum nomina sed eorum qui vel optime ediderunt vel plenissime, ut Corporum editores, superiores editiones enumersverunt; quae ipse descripseram asterisco numeris praefixo notavi - altera quae est critica et interpretatoria. Cuius primo loco saepius indicavi saeculi notam plerumque ex indiciis epigraphicis, interdum ex indole epigrammatis repetitam. Qua in re quin saepe erraverim non dubito, neque mirabitur qui quanta incuria praeter atticos titulos pleraque ex lapidibus describi soleant noverit quique quam fallax sit usus auriumque indicium expertus erit. Tum variam lectionem adstruxi, ne hanc quidem plenam: meliora enim ubi praesto erant apographa deteriora ut par est abieci, ubi dubitationi locus videbatur relinqui cari ne quid omitterem. Interpres esse studui qusm brevissimus et quam rarissimus; neque enim quae ex continua epigrammatum lectione sponte explicantur tamquam in tironum usum usquequaque monui, eis fere contentus quae ad emendationem apta et necessaria essent. Hoc enim inprimis consilium secutus sum ut epigrammatum verba emendatiora quam adhuc legebantur proponerem, quod fieri potuit vel melioribus adscitis apographis vel deteriorum fide adcuratius examinata vel selectis doctorum virorum emendationibus. Et has quidem minime adfirmo omnes me cognitas habuisse, sed quod paucas memoravi hinc nolim reliquas me ignorasse conligatur; praetermisi enim quidquid inutiliter aut falso disputatum vel coniectum demonstrari poterat, ne supra modum libri moles augeretur neve vituperandi essent quos aut laudare mallem aut ne vituperio quidem dignos putarem. Praeter Boeckhium et Kirchhoffium quibus equidem laudandis non par sum felicissimi epigrammatum vindices atque emendatores extiterunt Fridericus Jacobs et Godofredus Hermann, alter horum studiorum omnino princeps, alter hoc potissimum nomine praeclarus quod etsi non ubique verum reperiret ubique tamen ad verum reperiendum viam monstraret. Privatim praeter v0s ipsos, Hermanne Vsener et Francisce Buecheler, qui haerenti mibi nunquam auxilium denegavistis, unum nanctus sum operis tamquam socium Vdalricum de Wilamowitz, qui et interpretando et emendando quid potuerit praestare 
sciunt qui admirabilem eius doctrinam noverunt, quid voluerit pro egregia eius erga me voluntate omnis fere pagina clamitat : illi igitur iucundum est etiam publice gratias agere singulares.

Ipse denique quam adcuratissime tituli verba transcribere studui ne orthographicis quidem mendis sublatis, iota muto quod dicitur adscripto nbi traditur adscriptum, subscripto ubi omissum est in lapide. Emendandi copiam ita mihi paravi ut varias et lapidariorum et describentium errandi cassas ipse cum in Graecia Italiaque olim biennium versarer satis expertus ubi fieri potuit diligenter litterarum similitudines rimarer, ubi ex apographorum indole fieri nequibat epigrammatum usum diu observatum sequerer; quo fretus usu saepius mutila restituere conatus sum, ut pristinae formae imaginem repraesentarem; quod qui audacius factum clamitent ignoscant ubi bene cessit opera, ubi male cessit corrigant ipsi.

Ceterum monito vix opus est quae ab aliis inventa sunt ea vel minutissima ad suum quaeque auctorem me rettulisse; ubi nomen non additur meo ipsius agi periculo scitote. Permulta minus quam volueram emendata et perpolita manserunt, quorum alis nisi novis adhibitis apographis emendari posse desperavi, alia si quis sagacior integro iudicio rem adgressus erit facili fortasse opera sanabuntur. Ego cui diu incubui thesauro tandem e manibus dimitto.

Relicum est ut addam quae additurum me supra promiseram.

2. Artificis nomen restituit Lolling Mittheil. des archaeol. Inst. in Athen I 175 'Aprotíwo II]ápios. Cf. quae secuntur.

Add. 4. S. Subscripta vidit Lolling l. 8. 8. haec:

$$
\text { 4TION : LAPI .... O. } 4 \text { E }
$$

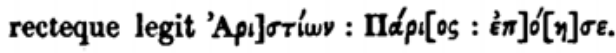

69, 1. lege ' $\Omega$ tòv $x \tau \lambda$ '.

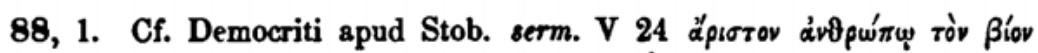

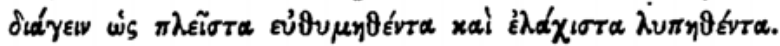

149. Litteris versuum initialibus nomen Sarapionem contineri vidit Nauck; ubi monuerit indicare nunc non queo. Intellege poetam. 
praefatio.

173, 21 sq. Agsthiae epigr. A. Pal. VII 551 :

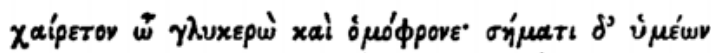

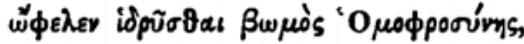

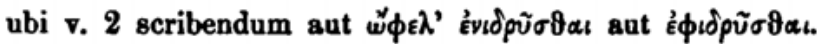

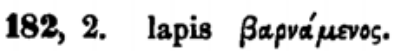

194. Sententia inde a v. 6 sic fere restituenda:

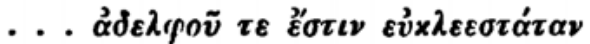

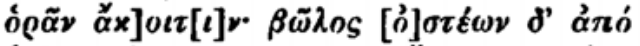

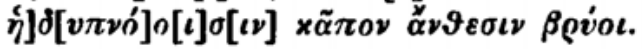

certum enim videtur verbum $\beta$ prév vi transitiva positum.

222b. Mileti. Rayet Revue archéol. 1874 XXVIII 113.

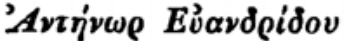

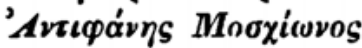

$$
\begin{aligned}
& \text { Xiovıs Xıóvıðog. }
\end{aligned}
$$

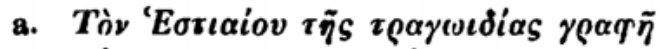

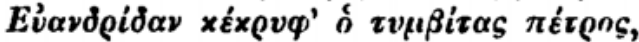

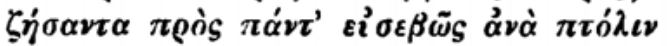

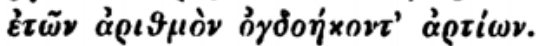

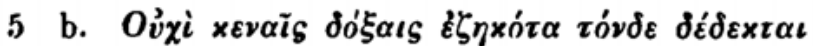

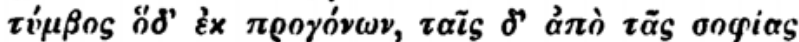

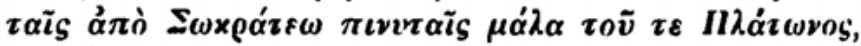

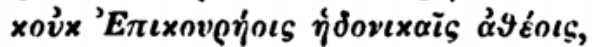

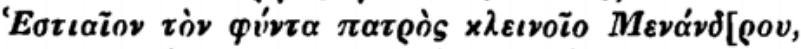

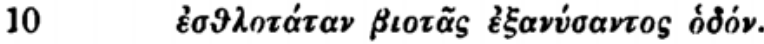

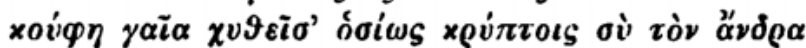

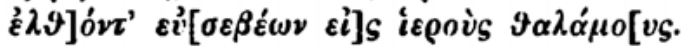

Circs Chr. natum videtur Antenor Eusndridae filius Hestiaei nepos incultos versus in patrem avumque scripsisse, alterum pridem alterum nuper sepulcro conditum. Nec tragicum poetam Euandridam nec Socraticnm novimus Hestiaeum; nomina ipsa testatur editor crebro in titulis Milesiis reperiri. - 1 articulum vocabulo $\tau$ parwdias adiectum ne mireris dixit Philostratus vit. soph. II 239 Clementem Byzantinum et $\tau \rho \alpha \gamma \omega$ días

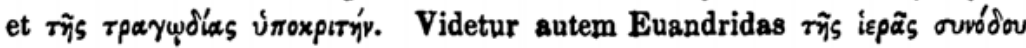




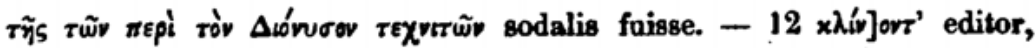
qui ceters recte supplevit. Pro ispor's cave conicias oxtepor's, cf. 222,8. 228,7 .

229 conl. Add. Ex ectypo ipse cognovi v. 1. Пw'גurta esse in lapide; idem nomen A. P. VII 335.

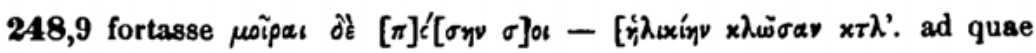
quod minus bene videbuntur proxima se adplicare inscitum adgnoveris interpolatorem, cf. adn.

251,1 sq. cf. Lconidae ep. A. P. VII 440 et index scriptorum 8. v.

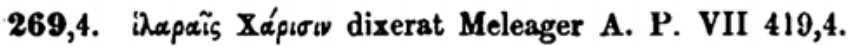

271. Falso haec interpretatus sum. Scriptum fuit epigramma sub Panis statua ab Ampelio una cum Musis in luco quodam posita: non

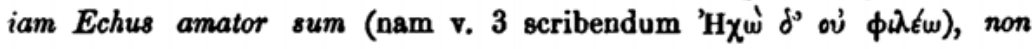
iam pastorum delector sodalicio, sed ab Ampelio huc translatus Musarum socius frigida fontium arborumque umbra fruor.

288 a. Cypro in insula. Ceccaldi Rev. archéol. 1875 I 97.

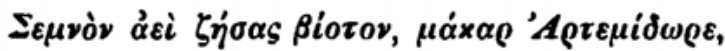

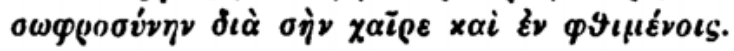

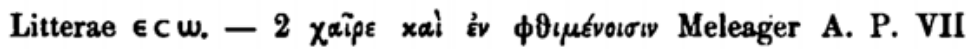
421,12; cf. epigr. sequens.

288 b. Cypro in insula. Ceccaldi Rev. archéol. 1875 I 97.

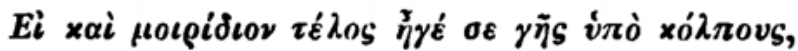

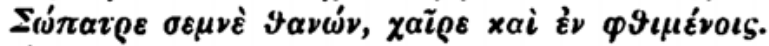

Litterae eaedem quae antecedentis tituli, eadem praeterea $\sigma \varepsilon \mu v^{\prime} \tau$ т laus, eadem denique clausula v. 2. 
288c. Cypro in insula. Ceccsldi Rev. arch, 1874 XXVII 94. $\dot{\eta} \varrho \pi \dot{\alpha} \sigma \vartheta[\eta \boldsymbol{\eta}$

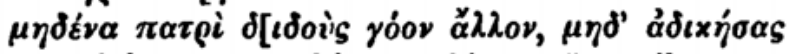

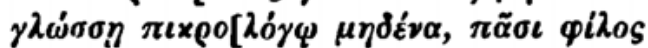

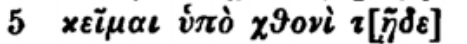

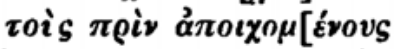

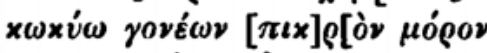

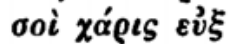

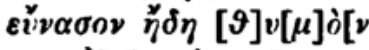

Litterae $\epsilon \boldsymbol{c} \boldsymbol{\omega} .-\mathbf{A b}$ initio traditur

PHIC HPIACEII

\section{MHAENATATPIS}

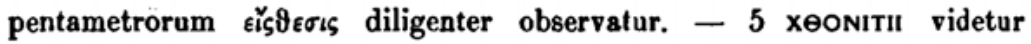
hereditario sepulcro conditus ad pridem mortuos proavos se contulisse dici. - 7 ronewn $P-8$ nescio cuius rei gratias agat Plutoni, ef. 462,14; fortasse precantem ( $\varepsilon^{\prime} \xi\left[\alpha^{\prime} \mu \varepsilon v 0 v\right]$ ) nescio quid deus exaudierat. - 9 нан YNO - 10 arKeTATPI rogatur deus iram missam faciat et adquiescat tribus quae rapuerit corporibus, cf. 413,7.

319,1. Eadem dictio $\mu \eta^{\prime} \mu \eta$ napadoûvaı est in Actis Timothei (ed. Vsener in progr. Bonnens. ad natal. inperat. 1877) p. 8.

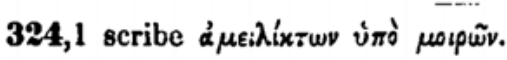

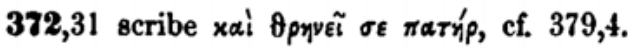

409,1 scribe Nopị́ üput Tpoxovdeús.

425,7 scribe $\sigma \tau \eta \lambda_{i ́ d \alpha} \chi \alpha \rho \alpha \xi \varepsilon v$.

431 a. Damasci. Waddington apud Lebas III part. VI n. 2549.

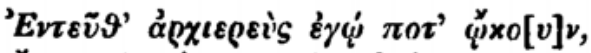

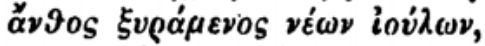

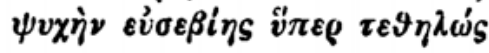




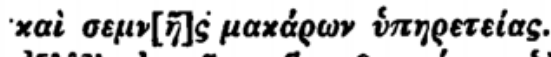

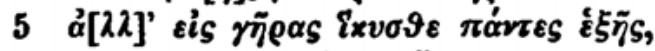

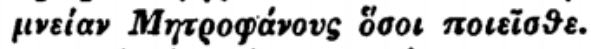

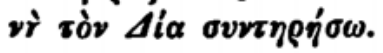

1 videtur ille ḱvraṽ̂a et ḱvreṽきv inter se confudisse. -2 sqq. barbam quidem tonsam, sed animum validum habui ad pium sanclumque deorum ministerium. - 4 cemnnic - ynнetenalc Buecheler Mus. $R h$.

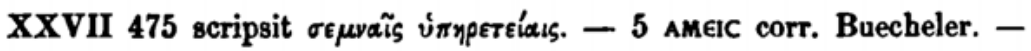
7 subscripsit pius homo idem.

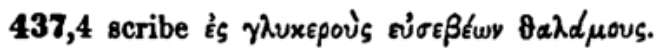

444,1 nomen fuerit 'Ovoparrós.

474 a. Spartae. Cumanudes Athenaei 1874 p. 484.

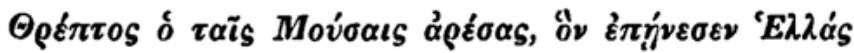

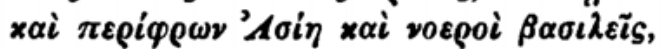

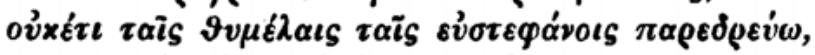

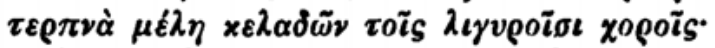

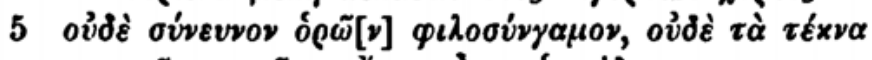

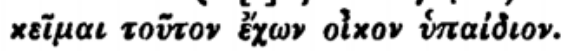

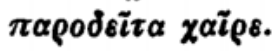

Aetatis inferioris esse dicitar Threpti tibicinis epitaphium; inperatores intellegere liset M. Aurelium et L. Verum (v. 2). -5 melius

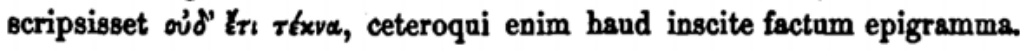

480 a. Mantineae. Foucart-Lebss 352 q.

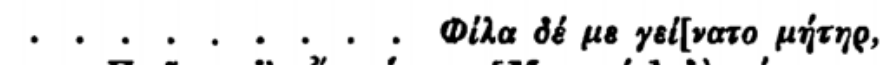

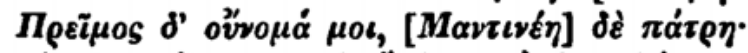

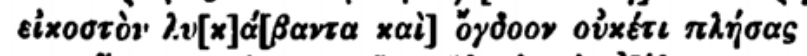

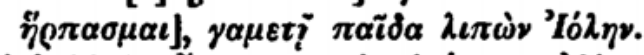

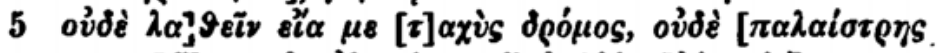

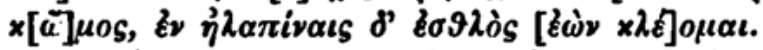

$\tau \alpha \tilde{v} \tau \alpha \mu \alpha \vartheta\left[\omega^{\prime}\right] \nu, \xi \xi \varepsilon \varepsilon, \pi \varepsilon i v e, \gamma\left[k \lambda \alpha, x \dot{\omega}^{\prime} \mu\right] \alpha \zeta \varepsilon, \mu \nu \rho i \zeta o v \cdot$

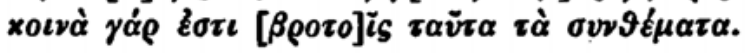

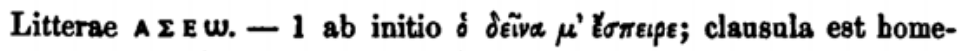

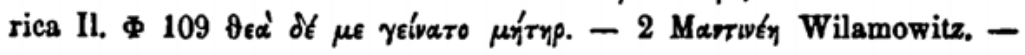


praefatio.

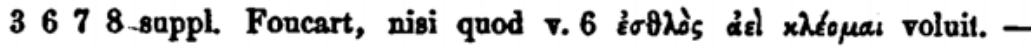
3 AYTA quod nescio an cum editore servandum sit. - or $\triangle O O N O N$ -

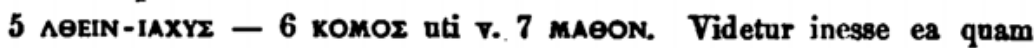
posni sententis haud ignobilem me sivit esse curous pernicilas aut turba palaestrae, cuirus laudabunt me sodales, cf. 663.

492. Ediderant Cumanudes Athenaei 1874 p. 478 et Foucart Rev. arch. 1875 I 110, qui v. 1 sqq. recte suppleverunt:

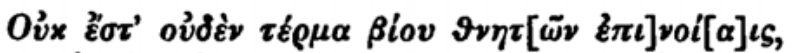

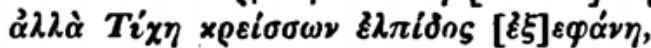

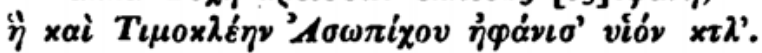

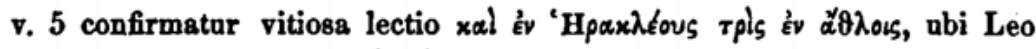

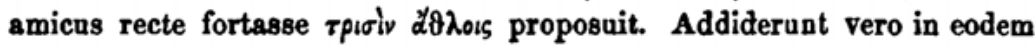
lapide ad dextram scriptum alterum epigramma, nimis quidem mutilum quam quod restitui possit, memorabile tamen non uno nowine:

$492 b$.

$$
\begin{aligned}
& \text { 'A }
\end{aligned}
$$

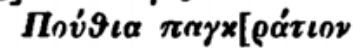

$$
\begin{aligned}
& \text { П } \alpha \tilde{\imath} \delta\left[\alpha M_{\varepsilon v] \varepsilon \times \rho \alpha \dot{\tau} \tau \varepsilon \omega}\right.
\end{aligned}
$$

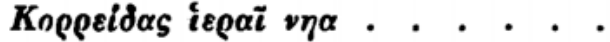

$$
\begin{aligned}
& \text { Ėvixas हैं }
\end{aligned}
$$

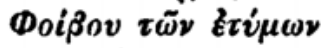

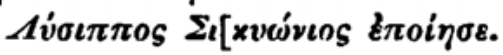

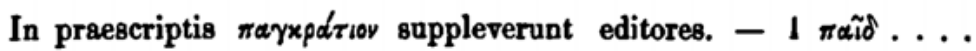
exparew Cum., kais.... aexpate tam litteram $P$ potius quam B sibi videri esse ait Foncart; qui deinceps IEPAINIA .. tradit; Cumanudes iєpã

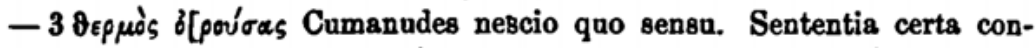

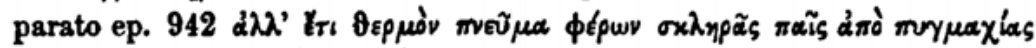

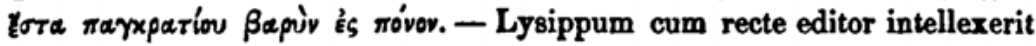
notissimum illum Sicyonium, Polycletum cuius nomen priori subscriptum est carmini non minus recte idem minorem adgnovit Lysippi aequalem, Naucydis discipulnm, ut iam certa babesmus saeculi quarti documenta. Notatn vero digna duorum epigrammatum eodem in lapide eodemque fere tempore insculptorum diversa dialectus.

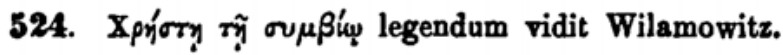




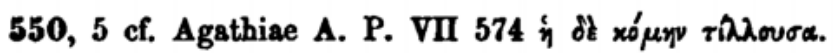

625, 3 vitium metricum sustuleris cum in archetypo pro $\pi 0 \lambda \lambda_{\alpha}^{\prime} \times 15$ fuisse sumpseris '̌́cáxı simile.

639. L. Arulenus Anoptes est C. I. L. V 5762.

640,4. Idem in eodem nomine lusus in ep. Antipatri Thessal.

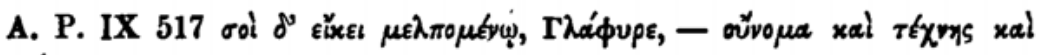
бw'matos.

646a. Romae. Garrucci Bullet. arch. 1861 p. 37 cum notis Kiesslingii et Detlefsenii.

$$
\Theta(\varepsilon \circ \tilde{\imath}) s[x(\alpha \tau \alpha \chi \vartheta o v i o \iota s) \text {. }
$$

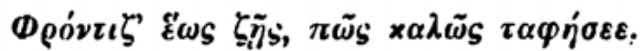

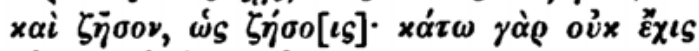

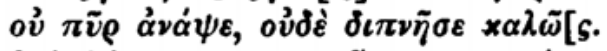

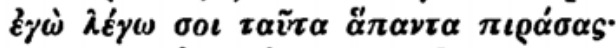

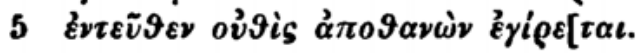

Litterse videntur $\in \mathrm{C} \omega$ fuisse. - 2 zнсоккAтs correxi; oี male Detlefsen. - 5 suppl. Kiessling.

647, 1 sq. cf. Senecae epitaphium in Anthol. lat. (ed. R.) 667 :

cura, labor, meritum, sumpti pro munere honores, ite, alias posthac sollicitate animas.

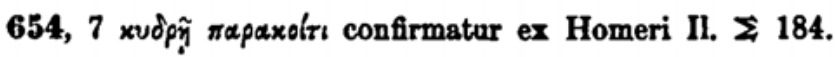

686, 5 sic fere scribendum videtur:

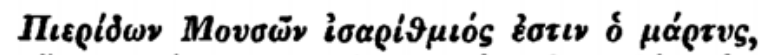

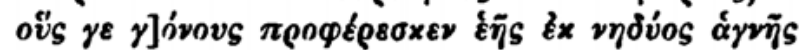

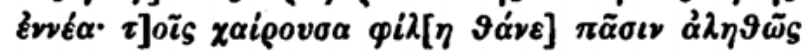

vitae testes etsi non ipsas Musas tamen liberorum numerum Musis aequalem habet, quos peperit novem.

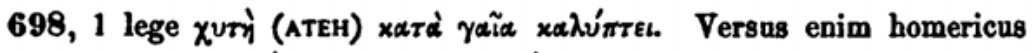

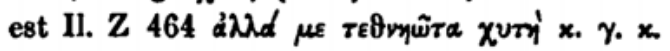




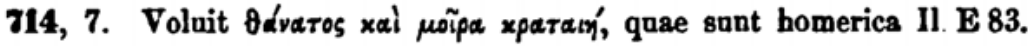

743 a. Athenis prope Olympieum. Bulletin de corréspondance Hellénique I 350. Ectypum vidi apud Kirchhoffium.

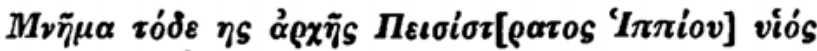

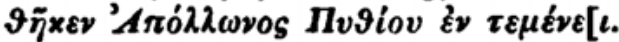

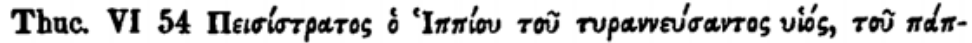

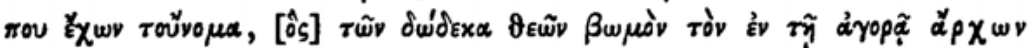

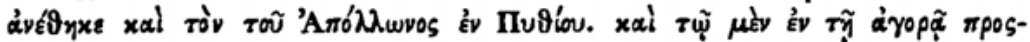

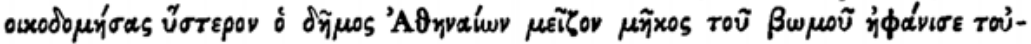

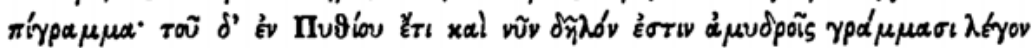

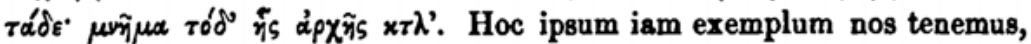
etsi cur á $\mu \nu \delta p \alpha '$ visa sint Thucydidi $\gamma p a ́ \mu \mu a \tau \alpha$ ex ectypo optimis clarissimis elegantissimis litteris scripto minime perspicitur. - 2 TEMENE iota lapide fracto periit.

757 a. Thisbe. Cumanudes Athenaei IV 378.

$$
\begin{aligned}
& \text { E } \\
& \text { है }
\end{aligned}
$$

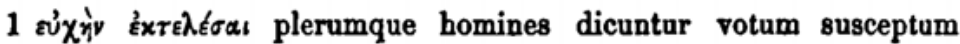
solventes.

768 a. Thebis. Bulletin de correspond. Hellénique I 351.

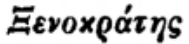

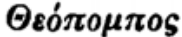

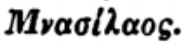

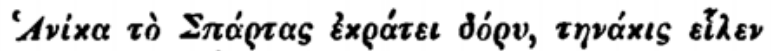

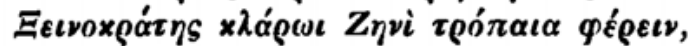

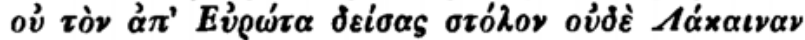

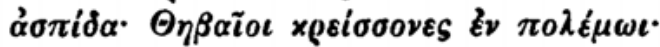

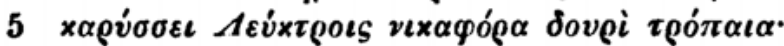

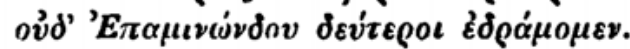

Narrat Pausanias IV 32,5 Thebanis cum instaret proelium Leuctricum a Trophonio inperatum esse ut clypeum olim ab Aristomene Messenio in herois fano consecratum inde repeterent tropaeique loco priusquam ad manus res veniret conlocarent; repetiisse autem Epami- 
nondae rogatn Xenocratem; quem clypeum conspicatos Lacedaemonios fugisse. Hanc Pausaniae narrationem cum epigrammatis memoria fere congruam esse intellexit Buecheler Mus. Rhen. XXXII 479 sq., ipsum epigramma non omni ex parte ut mihi videtar recte interpretatus. Sic intellego: dum Lacedaemonii in eo est ut vincerent (confirmatur igitur Xenophontis indicium Hellen. VI 4,13), Xenocrati sorto obtigit (sortiti autem erant praeter ipsum Theopompns et Mnssilaus) ut Iovi tropaeum adferret. Et adtulit ille hostium copias armaque nequaquam metuens: sic factum ut Thebani victores o pugna discederent, curus rei testimonium tropaeum est Leuctris positum. Princeps quidem victoriae auctor Epaminondas fuit (Diod. XV 56,2), nos vero propter utilem cursus celeritatem illo vix inferiores. Adparet ex his periculosum aliquod per medios hostes iter fecisse Xenocratem ad reciperandum illud quod Iovi poneretur tropaeum; adparet non minus (cf. v. 6) singulari quadam bac in re celeritate opus fuisse. Tropaeum quale fuerit") mirum est quod ex titulo non discimus (hastam enim fuisse hand recte Buecheler ex vv. 1 et 5 conlegit); sed et hoc et inscita narrandi ratio, dictionis inconcinnitas et obscuritas, dialecti inconstantia ( $\mathbf{} .5$ doupi) eo magis ducit ut epigramma non Iv saeculo sed tempore aliquanto recentiore scriptum putemus, quam eo ut rei memoriam a Pausania traditam fabulosa temporum longinquitate obscuratam dicamus. - 3 Diod. XV 53,2 oi de Bolwtol - xat-

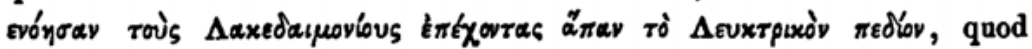
Xenocrati videtur percurrendum fuisse. - 5 vixaфópa doupl тpónaia sunt tropaes quibus victoria armis parta indicatur. - 6 currebant et Epaminondas et Xenocrates, alter contra hostes alter tropaeum ut repeteret.

784. Gravi meo errore factum est at epigrammatis huius et verbs et aetatem perperam constituerem. Teste enim Froehnero $\nabla$ a. Chr. n. saeculo scriptum est, legendum sutem sic:

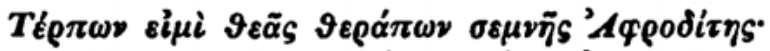

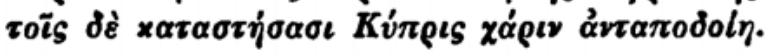

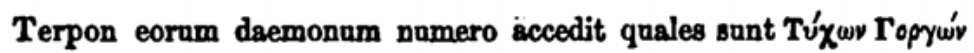
Kydadíwy alii.

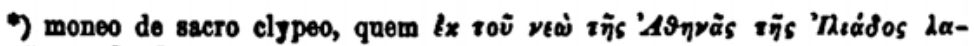

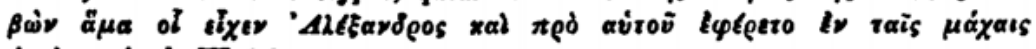
Arrian. Anab. VI 9,3.

Kalbel, Bplegrammata rracea. 
824 a. Tauromenii. Ex Camardae libro Lettera sulf epigrafe Taorminese (Palermo 1862) repetiit Bitschl $M, R h$ XXI 140 (Opusc. I 784).

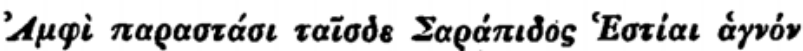

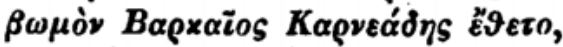

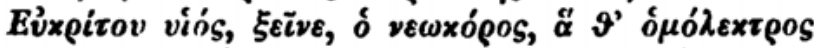

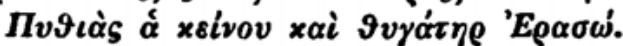

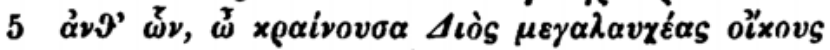

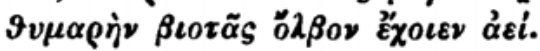

Litterarum ex formis Cavedoni (Atti e Memorie delle Deputazioni di storia patria per le provincie Modenesi et Parmesi Mod. 1863 I) probante Ritschelio titulum ante scriptum conlegit quam Augustus exactis incolis Tauromenio urbi praesidium romanum inponeret. - 2 Cyrenaeus fuit etiam Carneades philosophus cognominis.

-831 a. Romae. C. I. 5984 b.

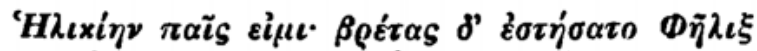

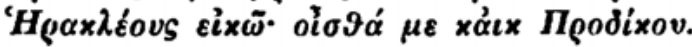

II fere p. Chr. n. saeculi.

831 b. Romae. C. I. 5972.

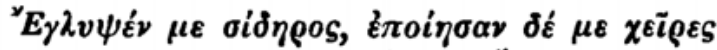

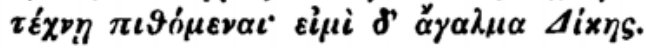

Litterae $\in$ C.

856 a. Hypate. Bulletin de corresp. Hellen. I 120 litteris minusculis.

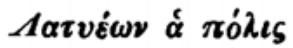

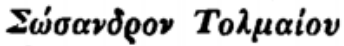

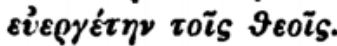

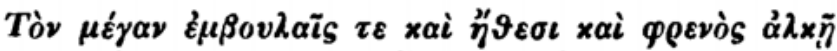

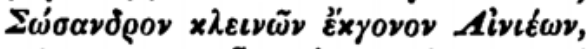

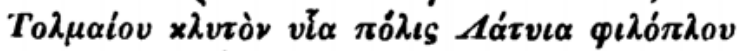

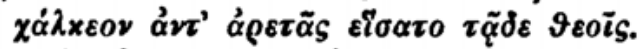

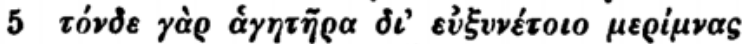

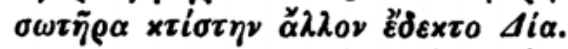

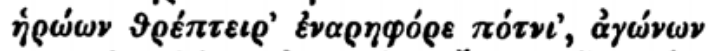

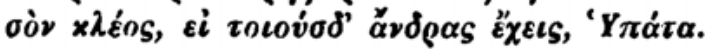


Litteras editor ait aetatis macedonicae esse; indoles carminis videtar recentior. - 2 Aivıĩ̄ formam pro es quae est vulgarior Aiviãves conmemoratam ab Eustathio n. B 749 adtulit editor. - 3 фihóntov gene-

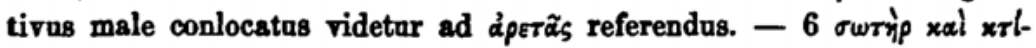

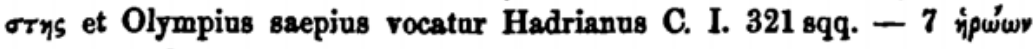
qualis est Sosander.

877b. Epidauri prope templum Aesculapii iuxta statuam virilem. Cumanudes Athenaei 1874 p. 273.

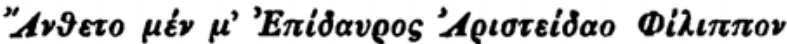

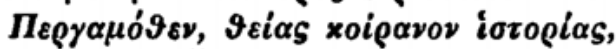

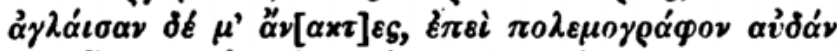

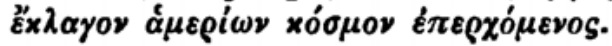

'E

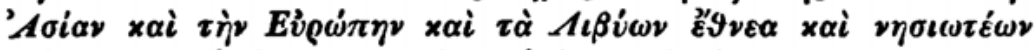

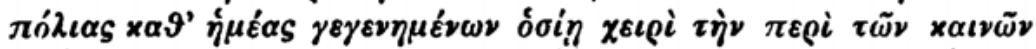

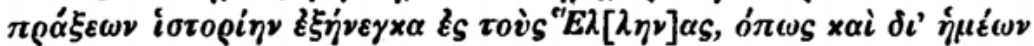

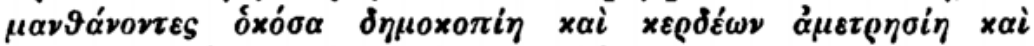

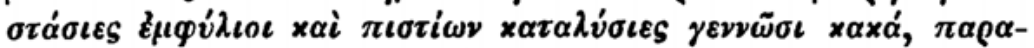

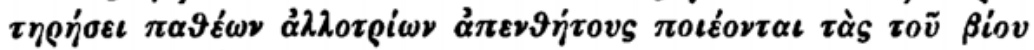
$\delta[v \varsigma \delta t] \rho \vartheta \omega \sigma i \alpha s$.

II p. Chr. n. saeculi. Ex eorum enim scriptorum numero est Philippus, quos Lucianus $\pi \tilde{\omega} s$ dei írop. ouryp. c. 16 sqq. dialecto ionics

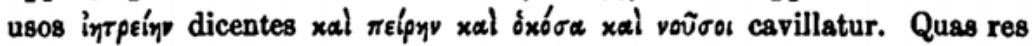
apud Afros insulanosque nuper gestas ille enarrasse se glorietur videmur ignorare. Epigramma civitas Epidauriorum, subscripta ipse Philippus insculpends cursvit. - Cumanudis lectionem bis correxi, et v. 3,

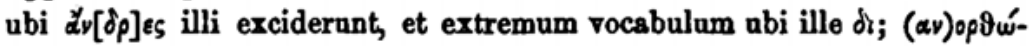
$\sigma$ sas edidit; intellege ut Graeci alienis malis cognitis ipsorum infortunia efficiant ut dolore careant.

931,1. $\mu \tilde{\eta} \lambda_{\alpha}$ Pythionicis praemia proposita saepius reperiuntur, cf. Luc. Anachars. c. 9.

932 a. Olympiae. Fráenkel Diar. arch. 1877 XXXV 47. ח

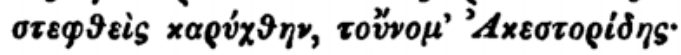


praofatio.

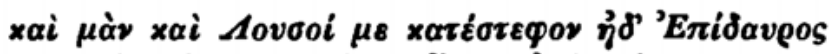

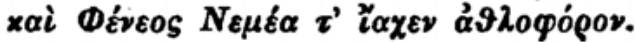

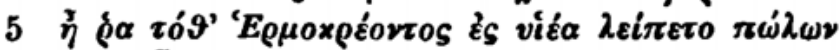

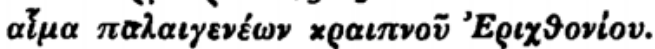

I fere a. Chr. n. saeculi. - 5 sententia non bene expressa haec est: tunc adparuit Erichthonï equorum prolem usque ad me Acestoriden Hermocreontis filium perdurasse. Equorum autem virtus (xpaınvótrs) haud magis eleganter io ipsum Erichthonium transfertur.

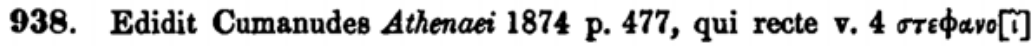
supplevit.

938 a. Thebis. Cumanudes Athenaei 1874 p. 477.

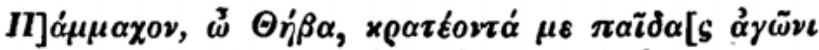

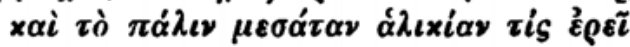

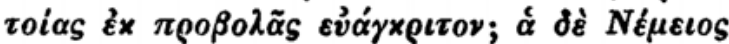

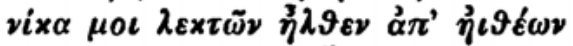

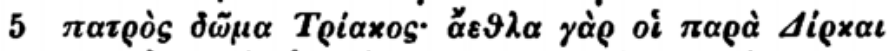

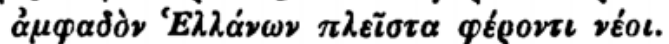

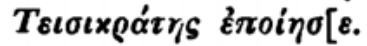

Tisicrates Sicyonius rv saeculi artifex. Eiusdem igitur aetatis Phorystae alterius Triacis filii epigramma (n. 938) est, unde corrige erro-

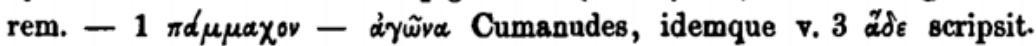
At sic procedit oratio: vici primum pueros, tum qui pueros inter iuvenesque aetate sunt medii, denique vero Nemea victoria (quae in causa fuit cur imago poneretur) ex iuvenibus reportata est.

942 a In provincia Trevisans repertum anaglypham. Veludo Atti dell Istituto Veneto di scienze lettere ed arti ser. III. vol. VII.

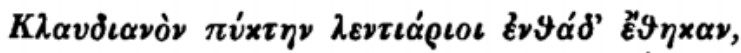

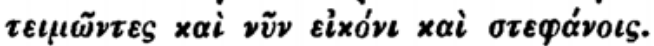

1 lintearii videntur ei vocari qui velorum in amphithestro curam agebant.

943 a. Athenis. Vssing Graeske og Latinske Indskrifter i Kjöbenhavn (1854) p. 24. 
praefatio.

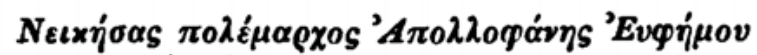

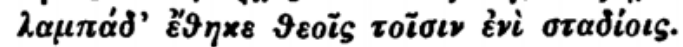

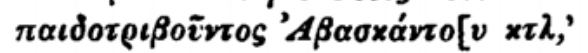

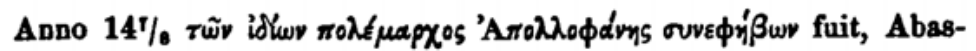
canti paedotribae anno decimo; cf. ad n. 960. -2 dei sunt Mercurius et Hercules, cf. n. 943,2.

1028. A se olim confectum apographum versunm 1-29 et 48-70 benigne mecum conmunicarit E. Curtius, ex quo haec enotabo: v. 11 XAPA

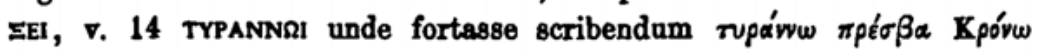

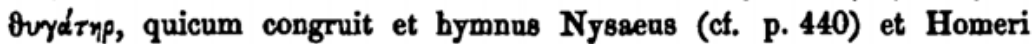

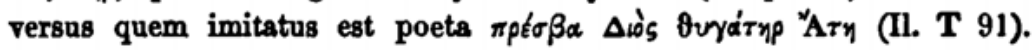
v. 16 enOTEY, v. 18 eainotponoze, v. 19 spanoz, v. 22 ENIETAzeI

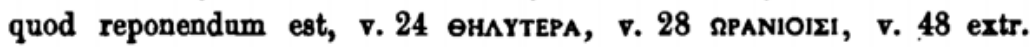

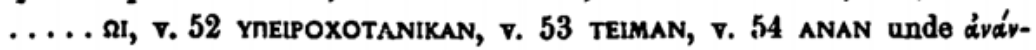
xav scribendum, จ. 62 En.

Eiusdem fere argumenti hymnum isiacum pedestri sermone scriptum apud Ietas inventum edidit Weil Mittheil. des arch. Inst. in Athen II 79, qui ab Eustratiade admonitus ridiculos suos de titalo errores ridicule retractavit ibid. II 189. Vtile erit Wilamowitzii adhibitis supplementis hymni verba huc transscribere.

octo fere versus perierunt ab initio

ż $\gamma \omega \dot{~} \varepsilon i \mu \iota . .$. . TYNAIKIOEOEKANOY

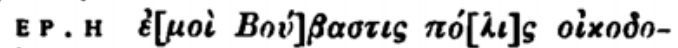

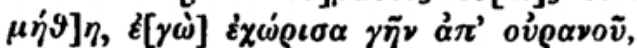

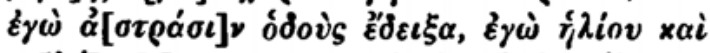

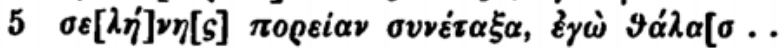

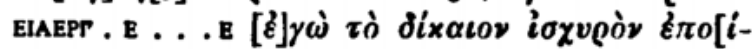

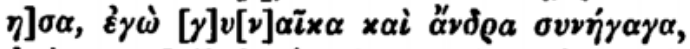

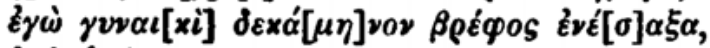

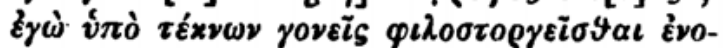

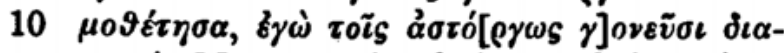

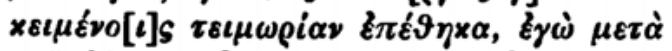

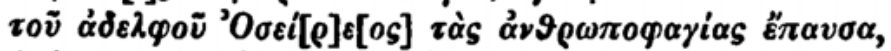

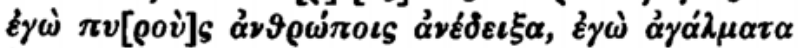

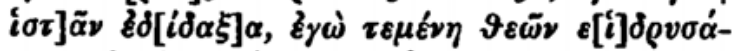

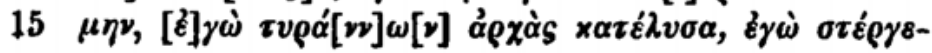


praefatio.

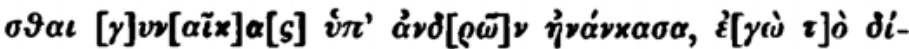

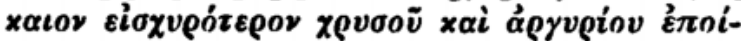

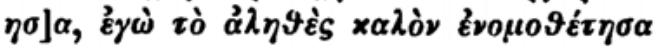

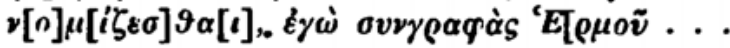

н... . . . TOY̌

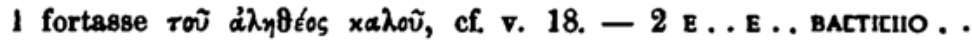
corr. Wilam. - 3 E.. IIEXWPILA corr. Weil. -4 A .... $\mathrm{N}$ corr. Wil.

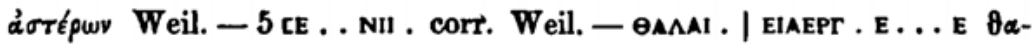

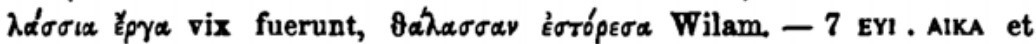
$8 \triangle$ EEKAI. INON corr. Weil. -8 ENEA 10 actoicomoneici corr. Wilam. - 12 oceneee corr. Wilam. - 13 urstric mupov's probabiliter Wilam. - 14 i $\sigma \tau \tilde{\alpha} \nu$ Wilam. $\dot{\varepsilon} \delta i \delta a \xi \alpha$ ego. EAPYCA - 15 kTWTYPAIW . corr. Weil. - 16 ceaIYN. . APYMANAIIIN et errosl corr. Weil. -17 xpufíov Wilam. -19 N.M.... eAt corr.

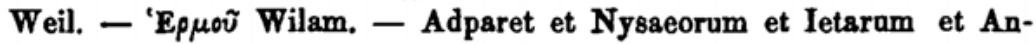
driorum hymnos ad unum omnes fontem referendos esse.

1044. Duo praeterea buins epigrammatis apographa extant, alterum Vischeri Archaeol. Beitr. tab. VI 1, alterum Foucarti recens editum apnd Lebas $331 \mathrm{a}$, quorum prior v. 2 inemerakneiaz legit, Foncart eodem v. extr. EYzebial, unde spretis Foucarti supplementis sic scribendum:

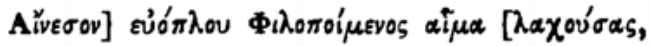

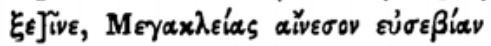

v. 3. Nano Vischer, v. 4 ANiaz . . N . KY $x \tau \lambda$ '. Foucart, qui recte

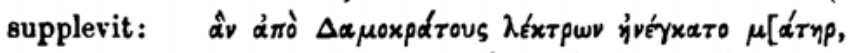

Ơंp]avias $[\sigma \varepsilon \mu] v[\alpha \cdot v] \mathbf{K} v$ inpudos iporódov.

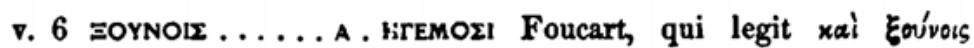

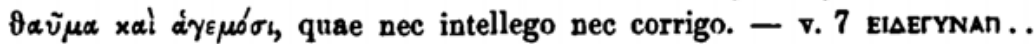
OIOKАA Vischer, extr. Foucart A. ZAтОфАMA - v. 8. yөАYMA Foucart, ... YnO .. ION. AI . . IAPET Vischer. Redintegrandum distichon aliis

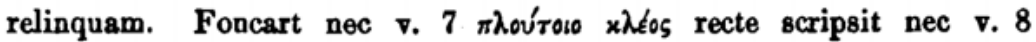
oú Oaupaбróv. Bene vere idem monuit Vraniae Veneris apud Megalopolitanos templum Psusanise aetate (VIII 35) iam dirutum fuisse.

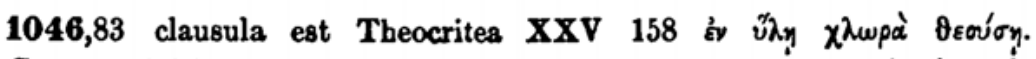
Ceterum initia versuum $31-46$ in fragmento marmoreo repetits invenit 
praefatio.

Detlefsen apud equitem Morbio Mediolanensem, praemisso fortasse no-

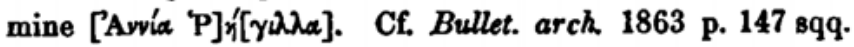

1046 a. Hermione. Foucart-Lebas $159 \mathrm{~g}$.

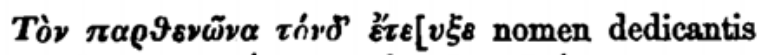

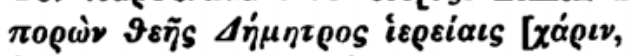

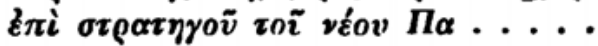

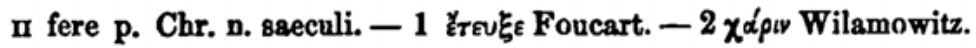

1072,5. Litterae entonton possunt fuisse eYtPonion, cf. v. 10.

1087. Epigramms expressum videtur ex [Aeschyli] ep. Bergk. Poet. Lyr. II 571 :

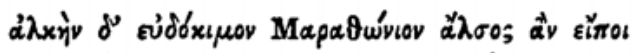

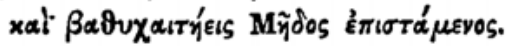

1100 a. Olympiae. Dittenberger Diar. arch. 1877 p. 96.

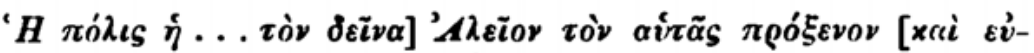

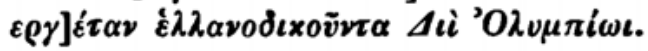

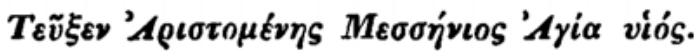

Ante Chr. n. factum videtur editori.

1117 a Tabula lignea ex Aegypto una cum sequenti in Americam translata, ubi extant in museo privato Eboracensi. Froehner Tablettes grecques du Musés de Marseillo (1867) p. 4.

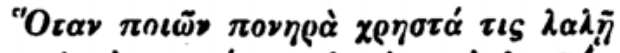

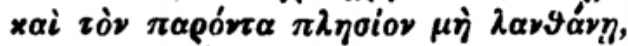

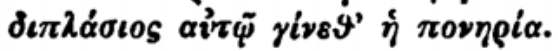

1117b. Tabula lignes; vid ad ep. anteced. Froehner l. 8. 8.

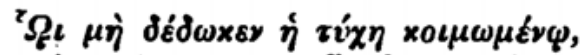

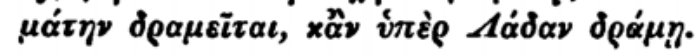

1119. Eosdem versus in quattuor tabellis similibus musei Borelli (ex museo Clot-Bey) legit Froehner Tablettes grecque du Musée de Marseillo p. 6, quarum in secunda tertia quarta praeteres haec sunt: M. Aujpy'hus

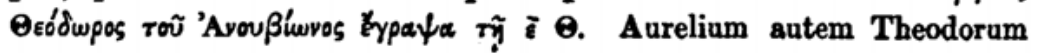


alia quaedam tabella et ipss ex Aegypto adlata docet fuisse saeculo in

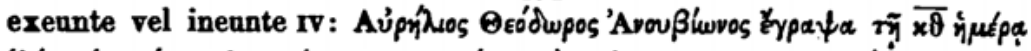

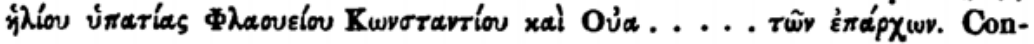
sules fuerunt Flavius Constantins et Galerius Valerins Maximinus annis 294, 300, 302, 305, 306; cf. Froehner l. 8. 8.

* 1119 a Diptychon Mediolani in museo Trivultiano. Mommsen C. I. L. $\mathbf{V} 8120$.

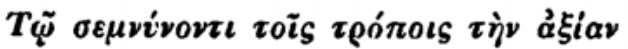

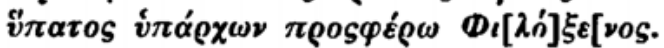

Fl. Theodo(rus) Filoxenus Sotericus Filoxenus vir ill. com. dom. ex magistr. per Thracia et consul ordin.

Anno p. Chr. n. 525. - 1 qui morum castitate consularem dignitatem inlustravit. -2 cf. seq. ep.

1119b. Diptychon Compendiense. Ex Gorio Mommsen C. I. L. V 8120 adn.

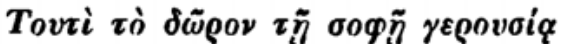

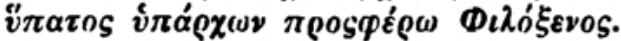

Vide ep. praeced.

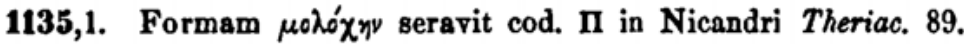

Additis quae habui addenda praefandi finem faciam. Penes vos autem, viri optimi, praeter ceteros iudicium esto; vobis si videatur hinc illinc bonum aliquod spicum ex bac segete legi posse, maximo opere gaudebo. Valete.

Scr. Roma Id. Decembr. a. h. s. LXXVII. 